\title{
Dependência da refração óptica não linear de origem térmica em nanofluidos de prata com a frequência da excitação óptica
}

Cristiano Santos Lopes Cristianofisico@gmail.com (UEPG), Ponta Grossa, Paraná, Brasil.

Vinicius Mariani Lenart vmlenart@gmail.com

de Ponta Grossa UEPG), Ponta Grossa, Paraná, Brasil.

Rozane de Fátima Turchiello Universidade Tecnológica Federal do Paraná (UTFPR), Ponta Grossa, Paraná, Brasil.

Sergio Leonardo Gómez

Universidade Estadual de Ponta Grossa (UEPG), Ponta Grossa, Paraná, Brasil.

\section{RESUM 0}

Coloides de nanopartículas de metais nobres e as suas propriedades físicas encontram aplicações práticas em numerosos campos, que vão da física à medicina, passando pela engenharia. Embora a sua síntese seja relativamente conhecida, as suas propriedades decorrentes da grande razão superfície/volume ainda apresentam desafios. Apresenta-se, neste artigo, um estudo experimental da dependência do índice de refração não linear $n_{2}$ de origem térmica de um coloide aquoso de nanopartículas de $\mathrm{Ag}$ com a frequência do obturador mecânico utilizado para gerar pulsos a partir de um feixe de luz laser continuo. 0 estudo foi feito empregando a técnica de Z-scan resolvida no tempo e mostra que um aumento da frequência leva a um aumento no valor de módulo de $n_{2}$, e um possível mecanismo é discutido.
\end{abstract}

PALAVRAS-CHAVE: Nanopartículas de prata; Índice de refração não linear; Lente térmica; Varredura z. 


\section{INTRODUÇÃO}

A pesquisa sobre propriedades ópticas de nanopartículas (NPs) de metais nobres têm merecido crescente interesse em aplicações como limitadores ópticos (RASHIDIAN et al., 2015), terapia fototérmica, imagens e biossensores (JAIN et al., 2008) entre outros.

As propriedades ópticas de NPs metálicas originam-se da ressonância do plasmon de superfície (SPR), uma oscilação coletiva dos elétrons de condução (M AIER, 2007). M etais nobres como Au, Ag e Cu exibem a banda de absorção do SPR na parte visível do espectro eletromagnético, cujos detalhes dependem de fatores como tamanho, forma e aglomeração das nanopartículas, e sobre as propriedades dielétricas do meio onde as nanopartículas são incorporadas (QUINTEM , 2011).

A origem das propriedades ópticas não lineares depende da escala de tempo do experimento. Para uma luz incidente durante intervalos de tempo da ordem de mili-segundos, a resposta óptica não linear é de origem térmica. Uma técnica muito simples e poderosa para estudar propriedades ópticas não lineares é a técnica Z-scan ou Varredura z (SHEIK-BAHAE et al., 1990), que emprega um único feixe para excitar e avaliar a resposta óptica não linear.

Sabe-se que a frequência de um laser pulsado está correlacionada com a resposta óptica não linear de um meio não linear devido ao efeito acumulativo da lente térmica (SWOFFORD e M ORRELL, 1978; GNOLI et al., 2005). Por outro lado, a dependência da frequência de corte da intensidade da lente térmica num coloide não foi suficientemente explorada. Nesta comunicação reportamos um estudo da dependência do índice de refração não linear de nanofluidos de prata com a frequência de iluminação da amostra. A técnica empregada foi a Z-scan com resolução temporal e os dados foram analisados utilizando o modelo de lente térmica.

\section{DETALHES EXPERIMETAIS}

\section{PREPARAÇÃO DAS NANOPARTÍCULAS DE PRATA}

O coloide aquoso de nanopartículas de Ag foi produzido adaptando o método originalmente proposto por Turkevich para a produção de nanopartículas de $\mathrm{Au}$ (TURKEVICH et al., 1951), por meio da redução do nitrato de prata $\left(\mathrm{AgNO}_{3}\right.$, Synth PA) com citrato de sódio dihidratado $\left(\mathrm{HOC}(\mathrm{COONa})\left(\mathrm{CH}_{2} \mathrm{COONa}\right)_{2} \cdot 2 \mathrm{H}_{2} \mathrm{O}\right)$, Synth PA) e polivinilpirrolidona (PVP, $\left(\mathrm{C}_{6} \mathrm{H}_{9} \mathrm{NO}\right)_{n}$, Sigma-Aldrich PA) como agente redutor e tensoativo, respectivamente. Estes reagentes foram utilizados sem qualquer purificação adicional.

A concentração do coloide obtido na síntese foi $(1,54 \pm 0,05) \times 10^{12}$ nanopartículas $/ \mathrm{ml}$. A partir do coloide inicial, amostras com concentrações menores foram obtidas por diluição com água duplamente destilada. Os valores das concentrações das amostras diluídas são indicados em porcentagem da concentração do coloide inicial. Para as medidas de Z-scan os nanofluidos foram acondicionados em porta amostras com caminho óptico de $200 \mu \mathrm{m}$, feitos de vidro de lâminas de microscópio. 


\section{A TÉCNICA DE Z-SCAN}

A técnica de Z-scan explora a indução de uma lente em um meio quando sobre ele incide um laser cuja intensidade possue perfil espacial Gaussiano $\left(T_{E M}{ }_{00}\right)$. Para um meio com absorção óptica não desprezível no comprimento de onda da luz incidente, o índice de refração correspondente pode ser escrito como $n=n_{0}+(d n / d T) \Delta T$, onde $n_{0}$ é o índice de refração linear, $d n / d T$ é o coeficiente termo-óptico e $\Delta$ T é o incremento na temperatura (BIALKOWSKI, 1996).

Durante a passagem do feixe de luz Gaussiano através da amostra, a luz é absorvida, induzindo 0 aquecimento local por relaxamento não radiativo com um perfil aproximadamente parabólico (GORDON et al., 1965) e um tempo característico da ordem de milissegundos. Geralmente, a luz transmitida pela amostra tem artefatos que surgem de efeitos ópticos lineares tanto da amostra como do porta amostra. Um exemplo é o espalhamento da luz produzido por imperfeções que possa ter o vidro com o qual é confeccionado o porta amostra.

Oliveira e Zlio propuseram realizar uma varredura de Z-scan com resolução temporal para limpar o sinal das contribuições espúrias (OLIVEIRA e ZLO, 1994). A transmitância normalizada $\Gamma_{\mathrm{N}}$ da amostra ao longo do eixo de propagação (eixo $z)$ em função da sua posição $z$ e após um tempo de irradiação t é definido por $\Gamma_{N}(z, t)=S(z, t) / S(z, t=0)$, onde $S(z, t)$ é o sinal do fotodetector posicionado no campo distante e centrado no eixo de propagação. Para um meio com uma resposta óptica não linear de origem térmica não muito intensa, $\Gamma_{N}(z, t)$ é dada por (CUPPO et al., 2002):

$$
\Gamma_{\mathrm{N}}(\mathrm{z}, \mathrm{t})=\left\{1+\left[\frac{\theta}{1+\frac{\left(1+\mathrm{x}^{2}\right) \mathrm{t}_{\mathrm{c} 0}}{2 \mathrm{t}}}\right] \frac{2 \mathrm{x}}{1+\mathrm{x}^{2}}\right\}^{-1},
$$

onde $\theta$ é o parâmetro do ajuste e está relacionado com a intensidade da formação da lente térmica, dado por:

$$
\theta=\frac{\alpha_{0} P d}{\kappa \lambda} \frac{d n}{d T}
$$

em que $d$ é o comprimento da amostra, $P$ é a potência do feixe, $\kappa$ é a condutividade térmica, $\alpha_{0}$ é a absorção óptica linear, $x=z / z_{0}, z_{0}$ é o comprimento de Rayleigh do feixe, $t_{c o}=w_{0}{ }^{2} / 4 D$ é o tempo de difusão característico, $D$ é a difusividade térmica e $w_{0}$ é a cintura do feixe. Um meio caracterizado por $\mathrm{dn} / \mathrm{dT}>0(\mathrm{dn} / \mathrm{dT}<0)$ comporta-se como uma lente convergente (divergente). 0 sinal e a magnitude de $\mathrm{dn} / \mathrm{dT}$ podem ser obtidos inequivocamente por meio da técnica de Z-scan de coletando a intensidade por uma íris de pequena abertura posicionada ao longo da direção de propagação do feixe após a amostra e longe desta, como mostrado na Figura 1.

A Equação 1 pode ser utilizada para dois tipos de medida, o ajuste da evolução temporal da transmitância, $\Gamma_{\mathrm{N}}(\mathrm{t})$, no estudo da formação da lente térmica, para um valor fixo de $z$, e também para uma medida de $Z$-scan, $\Gamma_{\mathrm{N}}(\mathrm{z})$, considerando um valor fixo para t.

0 coeficiente $\theta$ está relacionado ao índice de refração não linear $n_{2}$, presente na expressão do índice de refração: $n=n_{0}+n_{2} l$, segundo a expressão 


$$
\mathrm{n}_{2}=\frac{\lambda \varepsilon_{0} \mathrm{cn}}{2 \pi \mathrm{I}_{0} \mathrm{~d}} \theta,
$$

onde $\varepsilon_{0}$ é a permissividade do espaço livre (vácuo), c é a velocidade da luz no vácuo e $I_{0}$ é a intensidade do laser na cintura do feixe.

0 arranjo experimental inclui ainda um laser contínuo de $\lambda=473 \mathrm{~nm}$ (Ciel, Laser Quantum, 0,5 W), um obturador mecânico (chopper) (SR540, Standfor Research Systems) para a modulação do laser contínuo, um fotodetector de silício (818, Newport) e um osciloscópio digital (TDS1012B, Tektronix) com uma interface para aquisição de dados. Na figura tem-se: chopper (Ch), lente (L), amostra (S), íris (I) e fotodetector (D).

Figura 1 - Esquema da configuração experimental da técnica de Z-scan resolvida no tempo

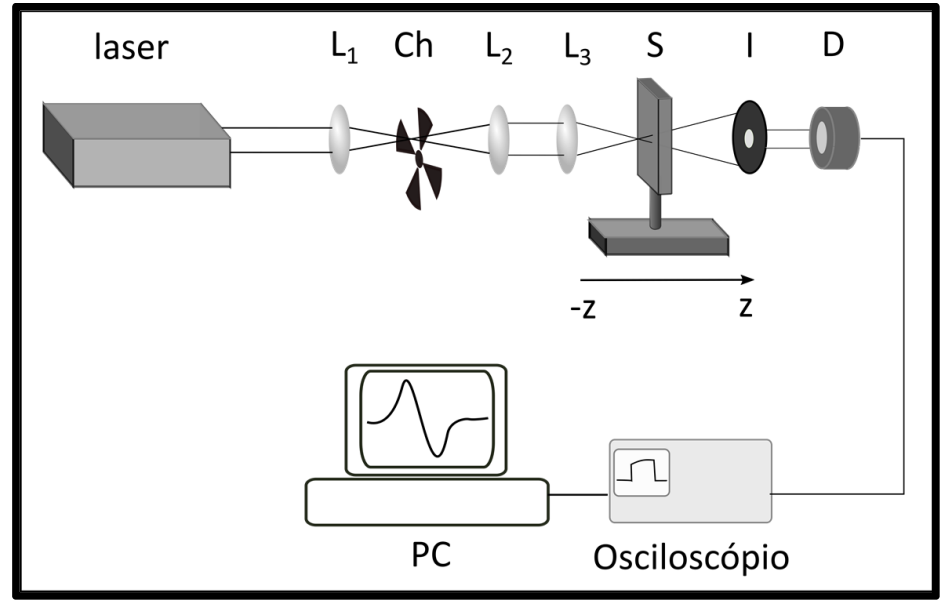

Fonte: autores.

A energia incidente sobre a amostra foi a mais baixa possível para evitar 0 aparecimento de efeitos hidrodinâmicos indesejáveis como, por exemplo, a convecção. A obturação do feixe de luz acontece no foco do conjunto de lentes confocais formado por $L_{1}$ e $L_{2}$, de modo a minimizar o tempo de subida do pulso de luz.

\section{RESULTADOSE DISCUSSÕES}

A Figura 2a mostra uma imagem por microscopia eletrônica de transmissão (TEM) das AgNPs com um tamanho médio de $32+10 \mathrm{~nm}$ e a Figura $\mathbf{2 b}$ mostra a distribuição de tamanho das nanopartículas obtida a partir da análise da imagen de TEM .

As medidas de Z-scan foram realizadas em função da concentração das amostras e da frequência do chopper. Todas as medições foram feitas com a intensidade do laser ajustados para valores tão baixos quanto possível para evitar efeitos espúrios e hidrodinâmicos indesejáveis como, por exemplo, a convecção. 
Figura 2 - a) Imagem por TEM das nanopartículas. 0 tamanho médio é aproximadamente 32nm; b) Histograma da distribuiç̧ão de tamanho

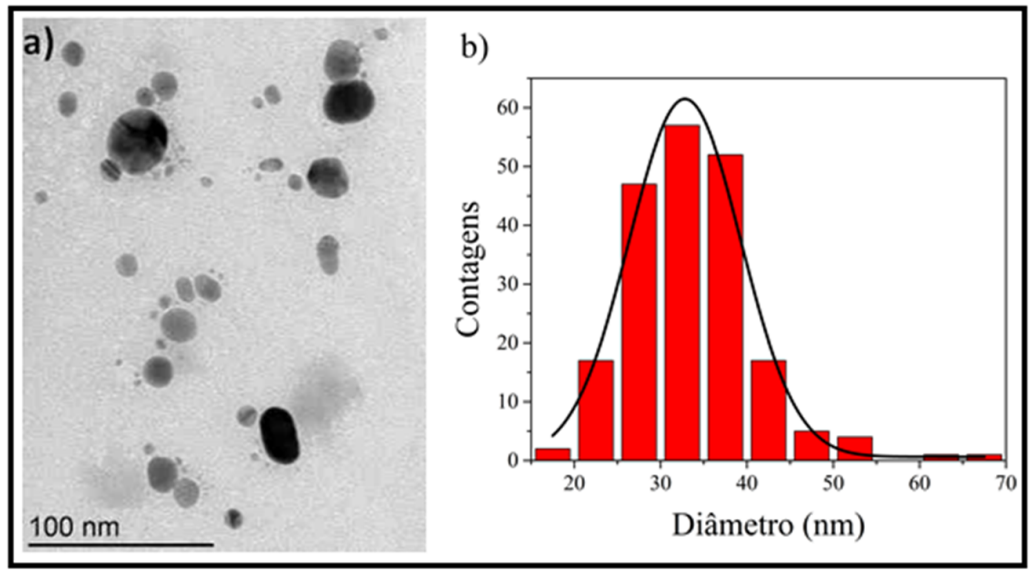

Fonte: autores.

A Figura 3 mostra o espectro de absorção das AgNPs. 0 espectro exibe a banda devida à ressonância do plasmon de superfície (SPR), atingindo um máximo próximo a $440 \mathrm{~nm}$. Ao aumentar a concentração, não há indícios de aglomeração o qual seria evidenciado por uma maior largura no espectro de absorção, conjuntamente com um deslocamento do máximo da absorção para 0 vermelho.

Figura 3 - Espectro de absorção UV-Vis para a amostra com 10\% da concentração inicial do coloide.

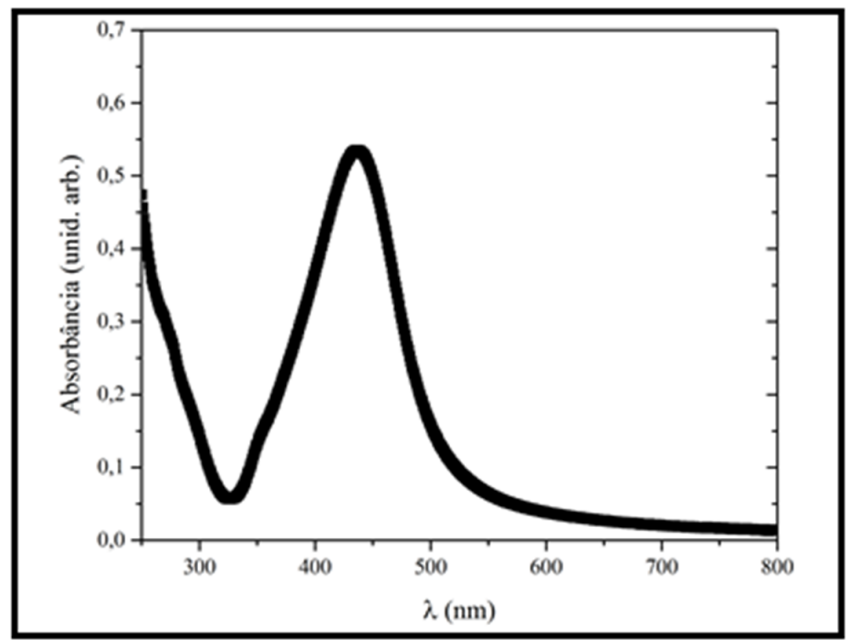

Fonte: autores.

A Figura 4 mostra a evolução temporal típica do sinal de lente térmica das amostras pesquisadas nesse trabalho, quando posicionadas antes do foco da lente $L_{3}$, quando sobre aquela incide um pulso retangular de luz. Para o caso da amostra estar posicionada após o foco da lente, a curva de evolução temporal seria a oposta, um sinal que decresce com o tempo. Este comportamento é típico de um sistema que se comporta como uma lente divergente. As amostras que se 
comportam como lentes convergentes apresentam comportamento oposto ao descrito anteriormente. A linha contínua mostra um ajuste à Equação 1.

Figura 4 - Curva experimental típica da evolução temporal do sinal de lente térmica para amostra posicionada antes do foco de $\mathrm{L}_{3}$

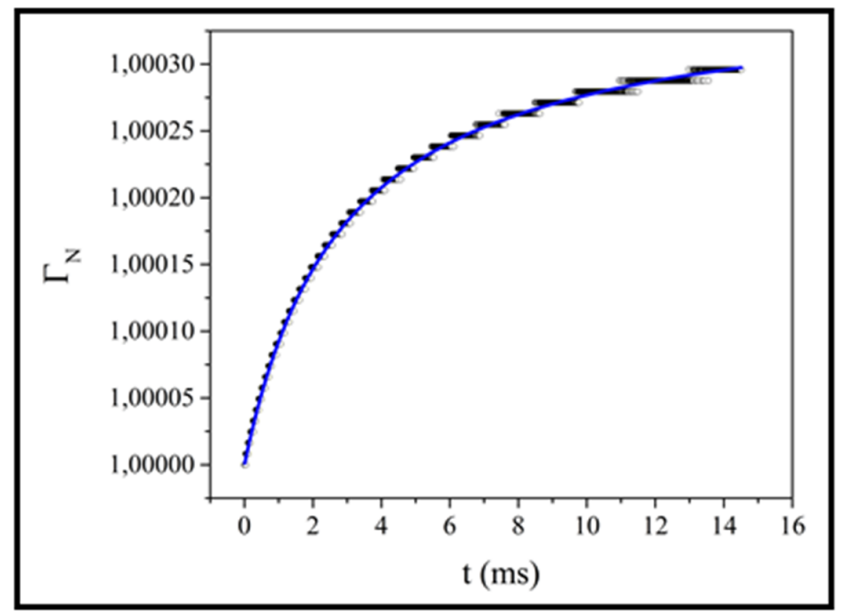

Fonte: autores.

A Figura 5 mostra uma curva típica de uma varredura Z-scan, obtida à temperatura ambiente para uma amostra de AgNPs. A linha contínua é o ajuste dos pontos com base na Eq. 1. As barras de erro correspondem ao erro padrão da média de, pelo menos, dez medições em cada posição z. Para as concentrações e potências utilizadas neste trabalho, o coloide exibe um efeito de autodesfocalização, ou seja, se comporta como uma lente divergente o qual corresponde a $d n / d T \ll 0$, e portanto $n_{2}<0$.

Figura 5 - Curva experimental típica de Z-scan correspondente a um [t] fixo

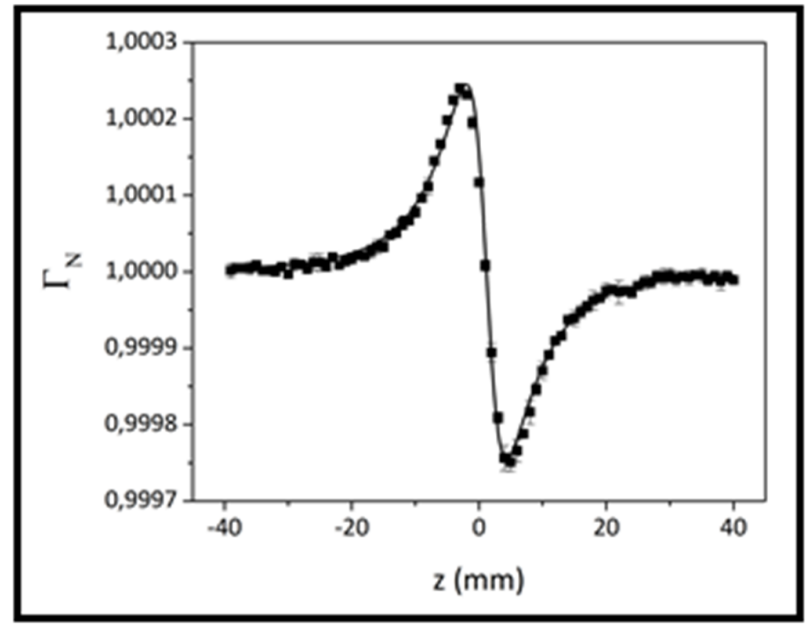

Fonte: autores.

Dentro da sensibilidade da nossa configuração experimental, e para as potências do laser utilizadas neste trabalho, as células de vidro vazias e o fluido portador das nanopartículas (água) não apresentam nenhum efeito de lente 
térmica. A absorção óptica não linear também não foi observada com os coloides. A linha contínua da figura mostra um ajuste dos dados à Equação 1.

A Figura 6 mostra o índice de refração não linear $n_{2}$ como uma função da concentração para duas frequências do chopper e para uma potência do laser incidente na amostra de aproximadamente $13 \mathrm{~mW}$. 0 aumento do valor absoluto de $n_{2}$ em função da concentração é esperado, pois $\theta$ e, portanto, $n_{2}$ dependem do coeficiente de absorção. Por outro lado, é possível observar também que, para uma concentração dada, $\mathrm{n}_{2}$ depende da frequência do chopper. 0 aumento da frequência do chopper leva a um aumento sistemático do valor absoluto de $\mathrm{n}_{2}$.

0 modelo teórico dado pela Equação 1 representa tanto a evolução temporal do sinal de lente térmica para uma posição z fixa da amostra, quanto a dependência em relação à posição z para um tempo t fixo de irradiação. Se somente um processo de difusão radial de calor estiver acontecendo, sem perda de calor na direção de propagação do feixe na amostra, os parâmetros do ajuste deveriam ser independentes da frequência da excitação óptica no intervalo de frequências considerado neste experimento.

0 resultado mostra que a suposição de não difusão de calor na direção longitudinal pode não ser correta para baixas frequências, já que o sistema teria maior tempo para dissipar calor através da superfície do portaamostras e, portanto o valor do coeficiente $n_{2}$ seria menor. A linha contínua é apenas para orientação.

Figura 6 - Gráfico de $n_{2}$ em função da concentração das AgN Ps para duas freqüências de corte diferentes

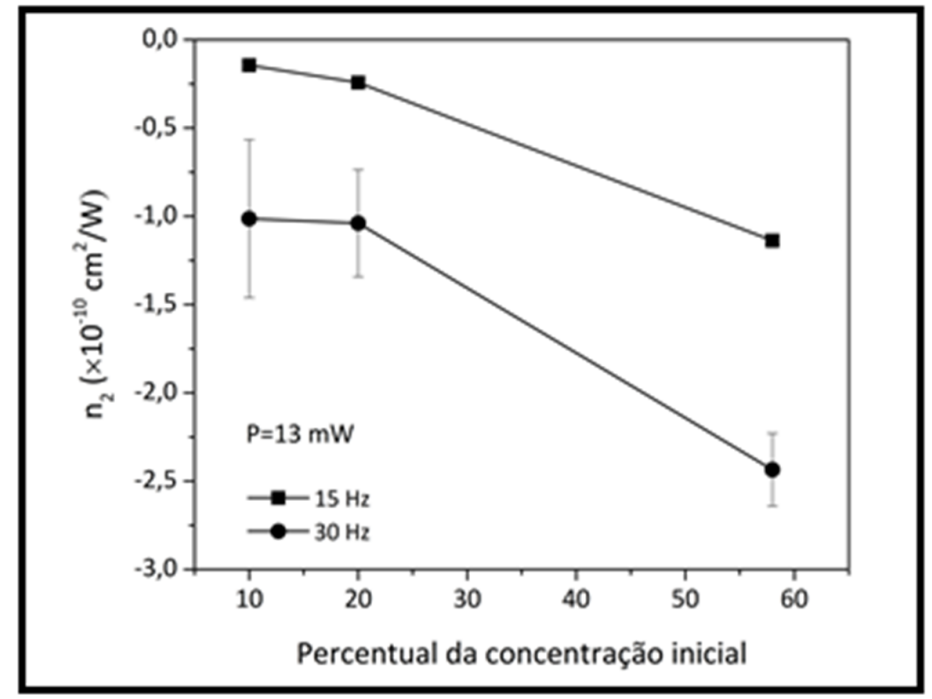

Fonte: autores.

A Figura 7 apresenta $n_{2}$ como uma função da frequência do chopper para diferentes concentrações do coloide e para uma potência do laser incidente de aproximadamente $14 \mathrm{~mW}$ e mostra claramente tanto um aumento do $\mathrm{n}_{2}$ em função da frequência quanto aparentemente uma tendência à convergência dos valores para baixas frequências, ou seja, maiores tempos de iluminação das amostras. 
Estudos prévios sobre a dependência de um sinal de lente térmica com a frequência da excitação óptica, utilizando pulsos de um laser continuo gerados por um chopper, em um experimento de lente térmica de configuração excitaçãoprova, mostrou que a intensidade da lente térmica diminui com o aumento da frequência de corte (BUFFETT e M ORRIS, 1983; RODRIGUEZ et al., 2014). A linha contínua é apenas para orientação.

Figura 7 - Gráfico de $\mathrm{n}_{2}$ em função da frequência de corte para três concentrações diferentes de AgNPs

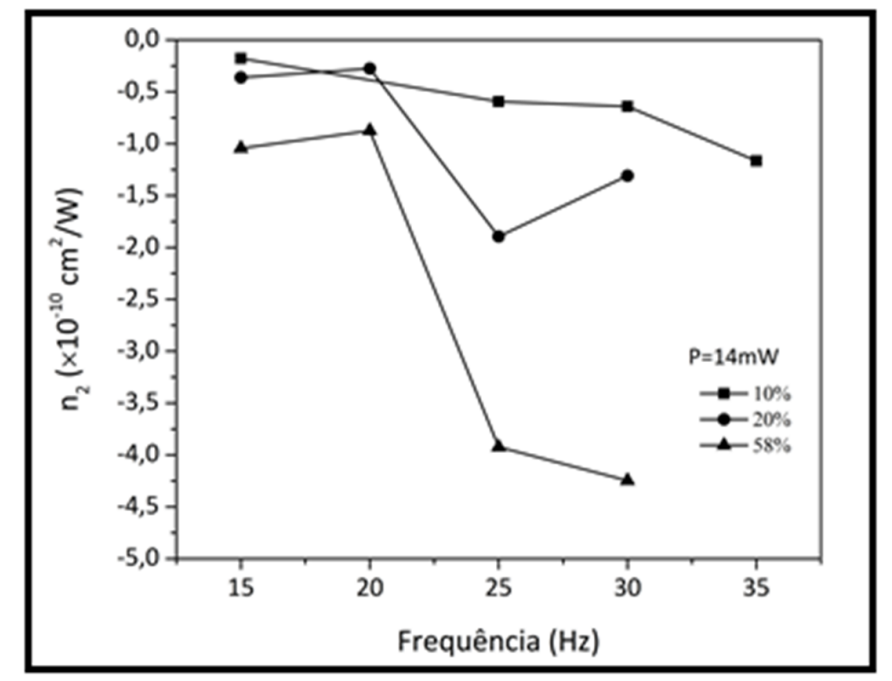

Fonte: autores.

Por outro lado, as medidas de $\mathrm{n}_{2}$ mediante a técnica de Z-scan utilizando baixas frequências do chopper podem ter os seus resultados influenciados por outros processos difusivos como, por exemplo, processos de difusão de matéria por gradiente de temperatura, conhecido como efeito Soret (ALVES et al., 2003; SEHNEM et al., 2016; KARIM ZADEH et al., 2013).

\section{CONCLUSÃO}

Nesta comunicação mostramos que na técnica de Z-scan com resolução temporal, usualmente utilizada para a adeterminação do índice de refração não linear de um meio, diferentes valores da frequência do chopper levam a diferentes valores do índice de refração não linear. Um aumento na frequência do chopper, leva a um aumento de $n_{2}$.

Uma possível origem do comportamento de $n_{2}$ em função da frequência poderia ser atribuído principalmente a uma contribuição de uma condução longitudinal do calor, com dissipação nas paredes dos porta amostra.

Outros processos difusivos, como o efeito Soret, podem ter uma contribuição no comportamteno observado de $n_{2} \times$ frequência. Assim, a escolha da frequência deve ser feita seguindo uma análise critériosa das condições de contorno e dos possíveis processos físicos que podem se apresentar. 


\title{
Dependencia de la refracción óptica no linear de origen térmico de nanofluidos de plata en función de la frecuencia de la excitacion óptica
}

\begin{abstract}
RESUMEN
Los coloides de nanopartículas de plata e sus propiedades físicas tienen aplicaciones en diferentes áreas, de la física a la medicina, pasando por la ingieneria. A pesar de que sus síntesis sean bien conocidas, las propiedades físicas que derivan del grande valor de la razón superficie/volumen todavía nos desafían. En esta publicación presentamos un estudio experimental de la dependencia del índice de refracción no linear $n_{2}$ de origen térmico de um coloide acuoso de nanopartículas de plata con relación a la frecuencia de un obturador mecánico, el cual es utilizado para producir pulsos a partir de un laser continuo. Este estudio fue realizado utilizando la técnica Z-scan con resolución temporal y muestra que el valor de $\mathrm{n}_{2}$ crece con la fruecuencia y um posible mecanismo es discutido.
\end{abstract}

PALABRAS CLAVES: Nanopartículas de plata; Índice de refracción no linear; Lente térmica; Escaneamento Z. 


\title{
Dependence of the thermal nonlinear optical refraction of $\mathrm{Ag}$ nanofluid on the chopping frequency of the optical excitation
}

\begin{abstract}
Colloids of nobre metal nanoparticules and their physical properties have applications in many fields from physics to engineering to medicine. Besides their syntheses are well known, the physical properties due to the large surface/volume ratio still defy us. In this work it is shown an experimental study of the dependence of the nonlinear refractive index $n_{2}$ of thermal origin of an aqueous colloid Ag nanoparticles as a function of the chopping frequency used to produce pulses from a CW laser. Such study, which was done by employing the time resolved Z-scan technique, showed that the absolute value of $n_{2}$ increases as a function of the frequency and a possible mechanism is discussed.
\end{abstract}

KEYWORDS: Ag Nanoparticles; Nonlinear refractive index; Thermal lens; Z-scan. 


\section{AGRADECIMENTOS}

Gostaríamos de agradecer o apoio financeiro das agências brasileiras CAPES, CNPq e Fundação Araucária e do Instituto Nacional de Ciência e Tecnologia em Fluidos Complexos (INCT-FCX).

\section{REFERÊNCIAS}

ALVES, S. et al. Investigation of the sign of the Soret coeffcient in different ionic and surfacted magnetic colloids using forced Rayleigh scattering and single-beam Z-scan techniques. Philosophical Magazine, v. 83, p. 2059-2066, 2003.

BIALKOWSKI, S. Photothermal Spectroscopy Methods for Chemical Analysis. Chichester: John Wiley \& Sons, 1996. Vol 134 da série Chemical Analysis: A Series of M onographs on Analytical Chemistry and its Applications.

BUFFETT, C. E.; MORRIS, M. D. Convective ffects in thermal lens spectroscopy. Applied Spectroscopy, v. 37, p. 455-458, 1983.

CUPPO, F. L. S. et al. Thermal-lens model compared with the Sheik-Bahae formalism in interpreting Z-scan experiments on lyotropic liquid crystals. Journal of the Optical Society of. America B, v. 19, p. 1342-1348, 2002.

GNOLI, A. et al. Z-scan measurements using high repetition rate lasers: how to manage thermal effects. Optics Express, v. 13, p. 7976-7981, 2005

GORDON, J. P. et al. Long-transient effects in lasers with inserted liquid samples. Journal of Applied Physics, v. 36, p. 3-8, 1965.

JAIN, P. K. et al. Noble Metals on the Nanoscale: Optical and Photothermal Properties and Some Applications in Imaging, Sensing, Biology, and Medicine. Accounts of Chemical Research, v. 41, p. 1578-1586, 2008.

KARIMZADEH, R. et al. Investigation of the Soret effect in copper nanoparticle colloids by self-phase modulation and Z-scan. Journal of Modern Optics, v. 60, p. 163-170, 2013.

MAIER, S.A. Plasmonics: Fundamental and Applications. New York: Springer, 2007.

OLIVEIRA, L. C.; ZILIO, S. C. Single-beam time-resolved Z-scan measurements of slow absorbers. Applied Physics Letters, v. 65, p. 2121-2123, 1994.

QUINTEN, M. Optical Properties of Nanoparticles Systems: Mie and Beyond. Weinheim: Wiley-VCH, 2011.

RASHIDIAN, M. et al. Thermo optical properties of Ag nanoparticles produced bypulsed laser ablation. Optical and Quantum Electronics, v. 47, p. 3729-3745, 2015.

RODRIGUEZ, L. et al. Measurement of thermal diffusivities of silver nanoparticle colloidal suspensions by means of a frequency-resolved thermal lensing approach. Optics Letters, v. 39, p. 3406-3409, 2014. 
SEHNEM, A. L. et al. Thermal Lens Phenomenon Studied by the Z-Scan Technique: Measurement of the Thermal Conductivity of Highly Absorbing Colloidal Solutions. Brazilian Journal of Physics, v. 46, p. 547-555, 2016.

SHEIK-BAHAE, M. et al. Sensitive measurement of optical nonlinearities using a single beam. IEEE Journal of Quantum Electronics QE, v. 26, p. 760-769, 1990.

SWOFFORD, R. L.; MORRELL, J. A. Analysis of repetitively pulsed dual-beam thermooptical absorption spectrometer. Journal of Applied Physics, v. 49, p. 3667-3674, 1978.

TURKEVICH, J. et al. A study of the nucleation and growth processes in the synthesis of colloidal gold. Discussions of the Faraday Society, v. 11, p. 55-75, 1951.

Recebido: 29 de abril de 2017.

Aprovado: 16 de junho de 2017

DOI:

Como citar: LOPES, C.S.; LENART, V.M.; TURCHIELLO, R.F. e GÓMEZ, S.L., Dependência da refração óptica não linear de origem térmica em nanofluidos de prata com a frequência da excitação óptica, Revista Brasileira de Física Tecnológica Aplicada, Ponta Grossa, v. 4, n.1, p. 72-83, mai./ jun. 2017.

Contato: Sergio Leonardo Gómez: sgomez@uepg.br

Direito autoral: Este artigo está licenciado sob os termos da Licença Creative Commons-Atribuição 4.0 Internacional.

(c) (1) 